\title{
Re: Incidences and Oncological Outcomes of Urothelial Carcinoma in Kidney Transplant Recipients
}

\author{
Yu J, Lee CU, Kang M, Jeon HG, Jeong BC, Seo SI, Jeon SS, Lee HM, Sung HH \\ Sungkyunkwan University Faculty of Medicine, Samsung Medical Center, Department of Urology, Seoul, Republic of Korea \\ Cancer Management and Research 2019:11;157-166 doi: https://doi.org/10.2147/CMAR.S185796
}

\section{EDITORIAL COMMENT}

The increased number of successfully performed kidney transplantations increases, life expectancy and the chance for urologists to treat urothelial carcinoma (UC) in kidney transplant (KTs) recipients. In this non-randomized retrospective single-center study, the authors aimed to investigate the incidence of UC by calculating age-standardized rates (ASRs) in 2186 KT recipients and the general population and to determine the treatment outcomes. ASRs of bladder cancer and upper urinary tract UC (UTUC) in KT recipients were 25.5 and 129.5 times higher than that in the general population and the proportion of female UC patients was 2.5 times higher, reflecting a reversed gender disparity. No significant difference was observed in treatment outcomes between KT recipients and nonKT patient, however, the rate of progression to bladder cancer was higher with a relative risk of $10.53(p=0.0481)$ compared to the general population. In the light of these data, urologic screening, and active treatment should be offered to identify the UC cases in KT recipients.

Yarkın Kamil Yakupoglu, MD

๑Copyright 2019 by the Association of Urological Surgery / Journal of Urological Surgery published by Galenos Publishing House. 